\title{
Compound Nevus
}

National Cancer Institute

\section{Source}

National Cancer Institute. Compound Nevus. NCI Thesaurus. Code C3901.

A nevus composed of neoplastic melanocytes that infiltrate both the epidermis and the dermis. 